\title{
Unified Analysis of a Class of Blind Feedforward Symbol Timing Estimators Employing Second-Order Statistics
}

\author{
Yik-Chung Wu and Erchin Serpedin
}

\begin{abstract}
In this letter, all the previously proposed digital blind feedforward symbol timing estimators employing secondorder statistics are casted into a unified framework. The finite sample mean-square error (MSE) expression for this class of estimators is established. Simulation results are also presented to corroborate the analytical results. It is found that the feedforward conditional maximum likelihood (CML) estimator and the square law nonlinearity (SLN) estimator with a properly designed prefilter perform the best and their performances coincide with the asymptotic conditional Cramer-Rao bound (CCRB), which is the performance lower bound for the class of estimators under consideration.
\end{abstract}

Index Terms - synchronization, timing recovery, feedforward, performance, analysis, Cramer-Rao bound.

\section{INTRODUCTION}

$\mathbf{T}$ HE PROBLEM of digital blind feedforward symbol timing estimation assumes recovery of the timing delay of the received signal based on the oversampled and unsynchronized received samples. Many algorithms were proposed in the literature to solve this problem. The earliest one is [1], in which the well-known square law nonlinearity (SLN) estimator is proposed. Several extensions of the SLN estimator were later reported in [2], [3] and [4], in which they consider more general second-order statistics than just squaring. Recently, the pre-filtering technique was applied to the SLN estimator to improve its performances at medium and high signal-to-noise ratios (SNRs) [5]. Other than square nonlinearity, estimators based on other types of nonlinearities were also proposed. Reference [6] proposed a logarithmic nonlinearity estimator, which is obtained by applying a low SNR approximation to the maximum likelihood (ML) method; absolute value and fourth order nonlinearities-based estimators were proposed in [7]; a combination of square and fourth order nonlinearities was proposed in [8] to take advantage of the properties of both types of nonlinearities. While all the above estimators require at least 3 samples per symbol, there are also some estimators that require only two samples per symbol. Reference [9] proposed such an estimator based on an adhoc argument. This estimator was later modified to remove its asymptotic bias in [10], and the pre-filtering technique was also applied to this estimator in [5] to improve its performance at medium and high SNRs. Reference [11] proposed another

Manuscript received August 11, 2004; revised October 9, 2004; accepted January 11, 2005. The associate editor coordinating the review of this letter and approving it for publication was A. Yener. This work was supported by NSF Award CCR-0092901.

The authors are with the Department of Electrical Engineering, Texas A\&M University, College Station, TX 77843-3128, USA (e-mail: \{ycwu, serpedin\}@ee.tamu.edu.

Digital Object Identifier 10.1109/TWC.2006.04007. two samples per symbol estimator based on the conditional maximum likelihood (CML) principle.

With so many estimators, designed using different philosophies and their performances analyzed independently under different assumptions, one would wonder whether we can have a general framework to analyze the performances of these estimators so that a fair and easy comparison can be made. This question was partially answered in [7], in which a technique for evaluating the jitter performance of symbol timing estimators employing a zero-memory, general type of nonlinearity was presented. In this letter, we analyze a different class of estimators (from that considered in [7]) by formulating all the blind feedforward symbol timing estimators employing second-order statistics (which include the estimators in [1], [2], [3], [4], [5], [9], [10] and [11]) into a single estimation framework, and then by deriving the finite sample (as opposed to the asymptotic performance reported in [8]) mean-square error (MSE) expression for this class of estimators. The MSE expression for any individual estimator can be obtained from the general expression by setting suitable parameters. The analytical results are compared with the computer simulation results, and it is found that both sets of results match very well. Furthermore, it is found that within the class of estimators employing second-order statistics, the SLN estimator with a properly designed prefilter and the feedforward CML estimator perform the best and their performances coincide with the conditional Cramer-Rao bound (CCRB) [12], which is the performance lower bound for the class of estimators under consideration.

The following notations are used throughout the letter. The symbols $\mathbf{x}^{*}, \mathbf{x}^{T}$ and $\mathbf{x}^{H}$ denote the conjugate, transpose and transpose conjugate of $\mathbf{x}$, respectively. Notation $\star$ stands for convolution. Notations $\mathfrak{R e}(x)$ and $\mathbb{E}[x]$ denote the real part and expectation of $x$, respectively. Matrices $\mathbf{I}_{N}$ and $\mathbf{0}_{M \times N}$ are the identity matrix with dimension $N \times N$ and the all zero matrix with dimension $M \times N$, respectively. Matrix $\mathbf{J}_{N}$ is the counter identity matrix, constructed by flipping $\mathbf{I}_{N}$ from left to right. $[\mathbf{Z}]_{i j}$ stands for the $(i, j)^{t h}$ element of matrix $\mathbf{Z}$. $\delta($.$) stands for the Kronecker's delta.$

\section{UNIFIED FORMULATION FOR THE}

Class of Symbol Timing Estimators Employing the SECOND-ORDER STATISTICS

For linear modulations transmitted through AWGN channels, the received signal can be written as shown in (1) at the top of the next page, where $\theta_{o}$ is the unknown phase offset; $E_{s}$ is the symbol energy; $d_{i}$ stands for the zero-mean unit variance, independently and identically distributed (i.i.d.) complex valued symbols being transmitted; $g(t) \triangleq g_{t}(t) \star g_{r}(t)$ 


$$
\left.r(n) \triangleq r(t)\right|_{t=n T_{s}}=e^{j \theta_{o}} \sqrt{E_{s} / T} \sum_{i} d_{i} g\left(n T_{s}-i T-\varepsilon_{o} T\right)+\eta\left(n T_{s}\right)
$$

is the combined response of the unit energy transmit filter $g_{t}(t)$ and the receiving filter $g_{r}(t) ; T$ is the symbol period; $T_{s} \triangleq T / Q$ with $Q$ being the oversampling ratio; $\varepsilon_{o} \in[0,1)$ is the unknown symbol timing delay to be estimated and $\eta\left(n T_{s}\right)$ stands for the samples of filtered noise. It is assumed that the noise samples before receive filtering is complex-valued circularly distributed Gaussian with power density $N_{o}$.

In this letter, we consider the class of estimators taking the following general form:

$$
\hat{\varepsilon}=-\frac{1}{2 \pi} \arg \left\{\sum_{k=0}^{K-1} \Lambda(k) e^{-j 2 \pi k / K}\right\},
$$

where $\Lambda(k)=\mathbf{r}^{H} \mathbf{B}_{k} \mathbf{r}$ with $\mathbf{r} \triangleq\left[r(0), r(1), \ldots, r\left(L_{o} Q-1\right)\right]^{T}$ is the observation vector of length $L_{o}$ symbols and $\mathbf{B}_{k}$ is a fixed matrix of dimension $L_{o} Q \times L_{o} Q$. The block diagram of this general estimator is shown in Fig. 1. After the observation vector is formed, $K$ samples of $\Lambda(k)$ are calculated, then a $K$-point discrete Fourier transform (DFT) is taken, and the phase of the first bin (i.e., second output) of the DFT scaled by a constant is the timing estimate. Let us now consider some special cases.

\section{A. Cyclic correlation-based estimator}

The cyclic correlation-based estimator [3], [8] is given by

$\hat{\varepsilon}=-\frac{1}{2 \pi} \arg \left\{\sum_{n=0}^{L_{o} Q-\tau-1} r^{*}(n) r(n+\tau) e^{-j \pi \tau / Q} e^{-j 2 \pi n / Q}\right\}$,

for $Q \geq 3$ and some integer lag $\tau \geq 0$. Note that different values of $\tau$ result in different previously proposed estimators in the literature ( $\tau=0$ corresponds to the estimators proposed in [1] and [4], $\tau=Q$ corresponds to the estimator in [2]).

If we decompose the summation term in (3) into $Q$ polyphase components and define $n_{u}(k) \triangleq\left\lfloor\left(L_{o} Q-\tau-1-\right.\right.$ $k) / Q\rfloor$, we have (4), as shown on the next page.

It can be seen that the cyclic correlation-based estimator takes the form of (2) with $K=Q$. Expressing $\Lambda_{C C}(k)$ into matrix form, we have $\Lambda_{C C}(k)=\mathbf{r}^{H} \mathbf{B}_{k}^{C C} \mathbf{r}$, where $\mathbf{B}_{k}^{C C}$ is a $L_{o} Q \times L_{o} Q$ matrix with its $(n Q+k, n Q+k+\tau)^{t h}$ element ( $\left.n=0,1, \ldots, n_{u}(k)\right)$ equal to $e^{-j \pi \tau / Q}$ and other elements equal zero. In particular, for the well-known SLN estimator [1] (i.e., $\tau=0, Q=4$ ), we have (for $i=0,1,2,3$ )

$$
\mathbf{B}_{i}^{S L N}=\mathbf{I}_{L_{o}} \otimes \mathbf{E}_{4}^{(i)}
$$

where $\otimes$ denotes the Kronecker product and $\mathbf{E}_{k}^{(i)}$ is a $k \times k$ matrix with the $(i, i)^{t h}$ element equals one and other elements equal zero.

\section{B. Lee's estimator and the modified estimator}

A two samples per symbol estimator was proposed by Lee in [9]. Later, this estimator was modified to remove its asymptotic bias [10]. The modified version of Lee's estimator can be written as (6), with $Q=2$ and $\gamma$ is a constant depending on the pulse shape $g(t)$. If $g(t)$ is a raised cosine pulse with roll-off factor $\rho$, then $\gamma=8 \sin (\pi \rho / 2) /\left(\rho \pi\left(4-\rho^{2}\right)\right)$ [10]. The original Lee's estimator can be obtained by setting $\gamma=1$. Now rewrite the expression in the $\arg \{\}$ of (6) as shown in (7).

Therefore, the estimator in (6) can also be expressed in the form of (2) with $K=4$. With the fact that $\mathfrak{R e}(x)=\left(x+x^{*}\right) / 2$ and expressing $\Lambda_{L e e}(k)$ in matrix form, we have $\Lambda_{\text {Lee }}(k)=$ $\mathbf{r}^{H} \mathbf{B}_{k}^{L e e} \mathbf{r}$, where the expression in (8) is true.

\section{Feedforward CML estimator}

The feedforward symbol timing estimator based on the conditional ML principle was proposed in [11]. Unfortunately, the results in [11] cannot be directly applied here since the original estimator was derived under the assumption that the noise samples are independent of each other, but in the signal model (1), the noise samples are correlated due to the receiver filtering. Of course, one can always start from the probability density function of $\mathbf{r}$, taking into account the correlation of noise and re-derive the estimator following the idea of [11]. However, a faster but equivalent method is as follows. Since the correlations between noise samples are related to the receiving filter (which is known), we can whiten the filtered noise samples by pre-multiplying the observation vector $\mathbf{r}$ with $\left(\varphi^{-1 / 2}\right)^{H}$, where $\varphi$ is the correlation matrix of the noise vector (with its elements given by $[\varphi]_{i j}=\int_{-\infty}^{\infty} g_{r}^{*}(t) g_{r}(t-$ $(i-j) T / Q) d t)$ and $\varphi^{-1 / 2}$ denotes any square root of $\varphi^{-1}$ (e.g., Cholesky decomposition) such that $\varphi^{-1 / 2}\left(\varphi^{-1 / 2}\right)^{H}$ $=\varphi^{-1}$. Then the results of [11] can be applied readily to this transformed observation vector $\left(\varphi^{-1 / 2}\right)^{H} \mathbf{r}$. It turns out that the resultant feedforward CML symbol timing estimator is given by $\hat{\varepsilon}=-\frac{1}{2 \pi} \arg \left\{\sum_{k=0}^{K-1} \Lambda_{C M L}(k) e^{-j 2 \pi k / K}\right\}$, where $K \geq 3$ and $\Lambda_{C M L}(k)=\mathbf{r}^{H} \mathbf{B}_{k}^{C M L} \mathbf{r}$ with variable $B_{k}^{M C L}$ defined in equations (9)-(11), and $L_{g}$ denotes the number of symbols affected by the inter-symbol interference (ISI) introduced by one side of $g(t)$. Notice that if $g(t)$ is bandlimited to $f= \pm 1 / T$ (an example of which is the raised cosine pulse), this feedforward CML estimator is valid for $Q \geq 2$. Notice also that if $g_{r}(t)=\delta(t)$, this estimator would reduce to the original proposed feedforward CML estimator in [11].

\section{Estimators with pre-filter}

In [5], a properly designed pre-filter was applied to the SLN estimator and the modified Lee's estimator to improve their performances at medium and high SNRs. In general, the prefiltering technique can be applied to the general estimator (2). In that case, the observation vector is composed of samples from the output of pre-filter. That is, $\Lambda_{P R E}(k)=\mathbf{x}^{H} \mathbf{B}_{k} \mathbf{x}$ with $\mathbf{x} \triangleq\left[x(0), x(1), \ldots, x\left(L_{o} Q-1\right)\right]^{T}$ and $x(n) \triangleq r(n) \star h(n)$ is the further filtered (apart from the receiver filtering) received signal samples through the pre-filter $h(n)$. If $h(n)$ is of finite length $L_{p}$, then $x(n)=\sum_{v=0}^{L_{p}-1} h(v) r(n-v)$ and the vector $\mathbf{x}$ is expressed as in (12). 


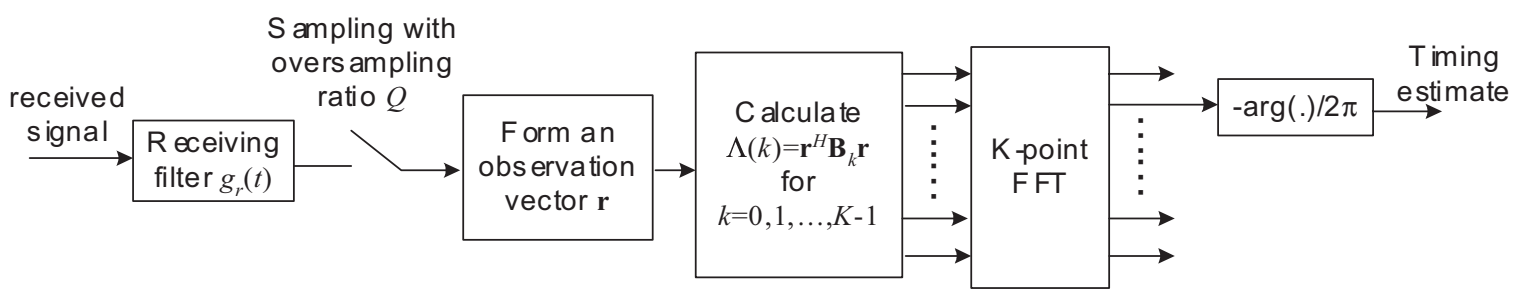

Fig. 1. Block diagram of the general estimator.

$$
\hat{\varepsilon}=-\frac{1}{2 \pi} \arg \{\sum_{k=0}^{Q-1} \underbrace{e^{-j \pi \tau / Q} \sum_{n=0}^{n_{u}(k)} r^{*}(n Q+k) r(n Q+k+\tau) e^{-j 2 \pi(n Q) / Q}}_{\Lambda_{C C}(k)} e^{-j 2 \pi k / Q}\} .
$$

$$
\hat{\varepsilon}=-\frac{1}{2 \pi} \arg \left\{\gamma \sum_{n=0}^{L_{o} Q-1}|r(n)|^{2} e^{j n \pi}+\sum_{n=0}^{L_{o} Q-2} \mathfrak{R e}\left[r^{*}(n) r(n+1)\right] e^{j(n-0.5) \pi}\right\},
$$

$$
\begin{aligned}
& \underbrace{\gamma \sum_{n=0}^{L_{o}-1}|r(n Q)|^{2}}_{\Lambda_{L e e}(0)}+\underbrace{\sum_{n=0}^{L_{o}-1} \mathfrak{R e}\left[r^{*}(n Q) r(n Q+1)\right]}_{\Lambda_{L e e}(1)} e^{-j \pi / 2} \\
& \quad+\underbrace{\gamma \sum_{n=0}^{L_{o}-1}|r(n Q+1)|^{2}}_{\Lambda_{L e e}(2)} e^{-j \pi}+\underbrace{\sum_{n=0}^{L_{o}-2} \mathfrak{R e}\left[r^{*}(n Q+1) r(n Q+2)\right]}_{\Lambda_{L e e}(3)} e^{-j 3 \pi / 2}
\end{aligned}
$$

$$
\begin{aligned}
& \mathbf{B}_{0}^{L e e}=\gamma \mathbf{I}_{L_{o}} \otimes \mathbf{E}_{2}^{(0)}, \quad \mathbf{B}_{1}^{L e e}=0.5 \mathbf{I}_{L_{o}} \otimes \mathbf{J}_{2}, \quad \mathbf{B}_{2}^{L e e}=\gamma \mathbf{I}_{L_{o}} \otimes \mathbf{E}_{2}^{(1)}, \\
& \mathbf{B}_{3}^{L e e}=\left[\begin{array}{ccc}
0 & \mathbf{0}_{1 \times 2\left(L_{o}-1\right)} & 0 \\
\mathbf{0}_{2\left(L_{o}-1\right) \times 1} & 0.5 \mathbf{I}_{L_{o}-1} \otimes \mathbf{J}_{2} & \mathbf{0}_{2\left(L_{o}-1\right) \times 1} \\
0 & \mathbf{0}_{1 \times 2\left(L_{o}-1\right)} & 0
\end{array}\right] \text {. }
\end{aligned}
$$

$$
\begin{aligned}
\mathbf{B}_{k}^{C M L} & \left.\triangleq \boldsymbol{\varphi}^{-1} \mathbf{A}_{\varepsilon}\left(\mathbf{A}_{\varepsilon}^{H} \boldsymbol{\varphi}^{-1} \mathbf{A}_{\varepsilon}\right)^{-1} \mathbf{A}_{\varepsilon}^{H} \boldsymbol{\varphi}^{-1}\right|_{\varepsilon=k / K}, \\
\mathbf{A}_{\varepsilon} & \triangleq\left[\mathbf{a}_{-L_{g}}(\varepsilon), \mathbf{a}_{-L_{g}+1}(\varepsilon), \ldots, \mathbf{a}_{L_{o}+L_{g}-1}(\varepsilon)\right], \\
\mathbf{a}_{i}(\varepsilon) & \triangleq\left[g(-i T-\varepsilon T), g\left(T_{s}-i T-\varepsilon T\right), \ldots, g\left(\left(L_{o} Q-1\right) T_{s}-i T-\varepsilon T\right)\right]^{T},
\end{aligned}
$$

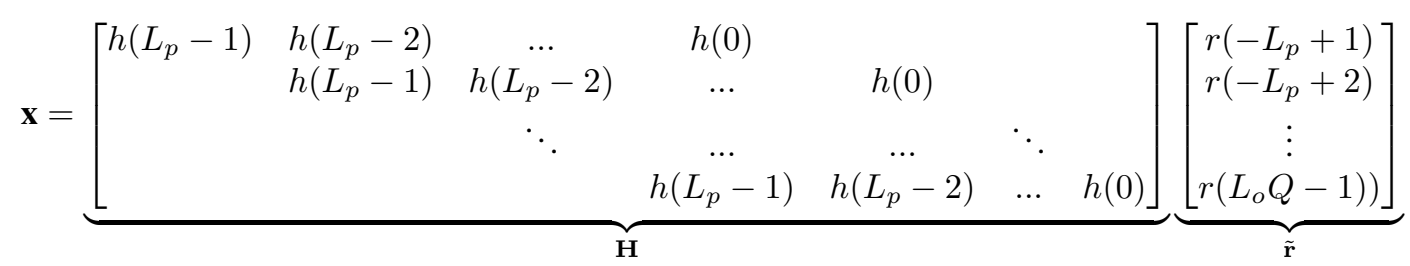


Therefore, the general estimator with pre-filter is $\hat{\varepsilon}=$ $-\frac{1}{2 \pi} \arg \left\{\sum_{k=0}^{K-1} \Lambda_{P R E}(k) e^{-j 2 \pi k / K}\right\}$, where $\Lambda_{P R E}(k)=$ $\tilde{\mathbf{r}}^{H} \mathbf{H}^{H} \mathbf{B}_{k} \mathbf{H} \tilde{\mathbf{r}} \triangleq \tilde{\mathbf{r}}^{H} \mathbf{B}_{k}^{P R E} \tilde{\mathbf{r}}$. For example, for the SLN estimator with pre-filter, we have

$$
\mathbf{B}_{k}^{P R E}=\mathbf{H}^{H} \mathbf{B}_{k}^{S L N} \mathbf{H} .
$$

Notice that, due to pre-filtering, although the observation vector $\mathbf{x}$ is of length $L_{o} Q$, the length of effective observation $\tilde{\mathbf{r}}$ (before pre-filtering) is $L_{o} Q+L_{p}-1$. Also, $\mathbf{B}_{k}^{P R E}$ is of dimension $\left(L_{o} Q+L_{p}-1\right) \times\left(L_{o} Q+L_{p}-1\right)$, rather than $\left(L_{o} Q-1\right) \times\left(L_{o} Q-1\right)$. Of course, if there is no pre-filter (i.e., $h(n)=\delta(n)$ ), all the equations in this subsection would reduce to that of the original estimator.

\section{Performance Analysis}

\section{A. Performance bound}

In [12], the asymptotic CCRB was introduced for symbol timing estimation problem. The asymptotic CCRB is a lower bound tighter than the modified Cramer-Rao bound (MCRB), but still a valid lower bound on the variance of any consistent estimator that is quadratic with respect to the received signal (which is the class of estimators under consideration). However, the asymptotic CCRB in [12] was derived assuming white Gaussian noise samples, therefore, the whitening technique similar to that in Section II-C has to be applied in order to include the effect of the receiving filter. Applying the results of [12] to the transformed observation vector $\left(\varphi^{-1 / 2}\right)^{H} \mathbf{r}$, it can be shown that for fixed $\varepsilon_{o}$,

$$
\mathrm{CCRB}^{a s}\left(\varepsilon_{o}\right)=\frac{1}{2 \operatorname{tr}\left(\tilde{\mathbf{D}}_{\varepsilon_{o}}^{H} \mathbf{\Psi}_{\varepsilon_{o}} \tilde{\mathbf{D}}_{\varepsilon_{o}}\right)}\left(\frac{E_{s}}{N_{o}}\right)^{-1}
$$

where $\tilde{\mathbf{D}}_{\varepsilon} \triangleq \frac{1}{\sqrt{Q}} d \mathbf{A}_{\varepsilon} / d \varepsilon$ and $\boldsymbol{\Psi}_{\varepsilon} \triangleq \boldsymbol{\varphi}^{-1}-$ $\boldsymbol{\varphi}^{-1} \mathbf{A}_{\varepsilon}\left(\mathbf{A}_{\varepsilon}^{H} \boldsymbol{\varphi}^{-1} \mathbf{A}_{\varepsilon}\right)^{-1} \mathbf{A}_{\varepsilon}^{H} \boldsymbol{\varphi}^{-1}$. Since the symbol timing delay $\varepsilon_{o}$ is assumed to be uniformly distributed in $[0,1)$, the average asymptotic CCRB can be calculated by numerical integration of (14). Notice that although the value of $Q$ appears in the expression of the asymptotic CCRB, numerical computations show that the asymptotic CCRB is independent of $Q$ as long as $Q \geq 2$. This is reasonable, since the asymptotic CCRB is derived under the assumption of white noise, oversampling the signal above Nyquist rate does not provide any new information, thus the ultimate performance cannot be improved.

\section{B. MSE expression}

In this section, we present the MSE expression for the general estimator (2). The derivation procedures follow closely to that in [11]. The only difference is that, the MSE expression in [11] was derived under the assumption of white noise, while in this letter, the correlation of noise has to be taken into consideration. This can be easily done by modifying just a few lines of the derivations in [11]. Due to space limitation, only the results are presented. Interested readers can refer to [11]. It can be shown that for a true timing delay $\varepsilon_{o}$, the MSE of the general estimator (2) is given by

$$
\operatorname{MSE}\left(\varepsilon_{o}\right) \triangleq \mathbb{E}\left[\left(\hat{\varepsilon}-\varepsilon_{o}\right)^{2}\right] \cong-\left(\frac{1}{2 \pi}\right)^{2} \frac{\mathfrak{R e}\left(\phi_{1}\right)-\phi_{2}}{\mathfrak{R e}\left(\phi_{1}\right)+\phi_{2}},
$$

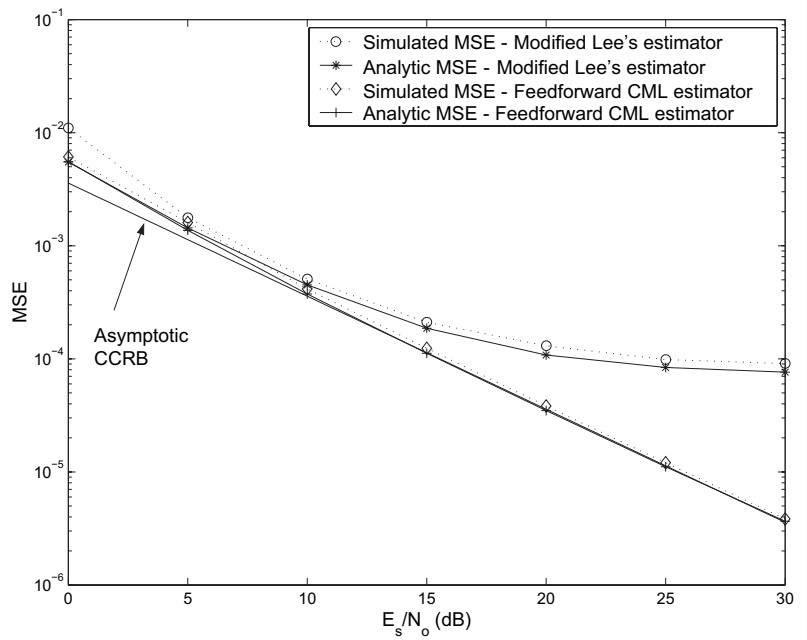

Fig. 2. Analytic and simulated MSEs for modified Lee's estimator and feedforward CML estimator.

where

$$
\begin{gathered}
\phi_{1} \triangleq e^{j 4 \pi \varepsilon_{o}} \sum_{k_{1}=0}^{K-1} \sum_{k_{2}=0}^{K-1} \mathbb{E}\left[\Lambda\left(k_{1}\right) \Lambda\left(k_{2}\right)\right] e^{-j 2 \pi k_{1} / K} e^{-j 2 \pi k_{2} / K}, \\
\phi_{2} \triangleq \sum_{k_{1}=0}^{K-1} \sum_{k_{2}=0}^{K-1} \mathbb{E}\left[\Lambda\left(k_{1}\right) \Lambda^{*}\left(k_{2}\right)\right] e^{-j 2 \pi k_{1} / K} e^{j 2 \pi k_{2} / K}
\end{gathered}
$$

In the above equations, the cross-correlation factors take the expressions given by equations (18), (19), and (21), where $\operatorname{tr}[$.$] denotes the trace of a matrix, \mathbf{R}_{\varepsilon} \triangleq \frac{E_{s}}{T} \mathbf{G}_{\varepsilon}+\frac{N_{o} Q}{T} \boldsymbol{\varphi}$,

$$
\left[\mathbf{G}_{\varepsilon}\right]_{i j} \triangleq \sum_{n=-\infty}^{\infty} g^{*}(i T / Q-n T-\varepsilon T) g(j T / Q-n T-\varepsilon T),
$$

with $\mathbf{a}_{n}\left(\varepsilon_{o}\right)$ defined in (11), and $m_{4}=\mathbb{E}\left[\left|d_{i}\right|^{4}\right]$ is the fourth order moment of the transmitted symbols, which is fixed for a specific constellation (e.g., $m_{4}=1$ for PSK and $m_{4}>1$ for QAM). As the symbol timing delay $\varepsilon_{o}$ is assumed to be uniformly distributed in $[0,1)$, the average MSE is calculated by numerical integration of (15). Notice that the expressions of $\mathbb{E}\left[\Lambda\left(k_{1}\right) \Lambda\left(k_{2}\right)\right]$ and $\mathbb{E}\left[\Lambda\left(k_{1}\right) \Lambda^{*}\left(k_{2}\right)\right]$ in (18) and (19) are more compact than the corresponding expressions presented in [11], but they are equivalent. This can be easily seen by the fact that if we expand the expressions in (18) and (19) and putting $\varphi=\mathrm{I}$, then we would obtain the corresponding expressions given in [11].

\section{NumERICAL EXAMPLES AND DisCUSSIONS}

In this section, the general analytical MSE expression presented in the last section will be plotted as a function of $E_{s} / N_{o}$ for different estimators. The analytic results are compared with the corresponding simulation results and the asymptotic CCRB. All the results are generated assuming i.i.d. QPSK data, $L_{o}=100$, both $g_{t}(t)$ and $g_{r}(t)$ are square root raised cosine pulses with $\rho=0.3, L_{g}=3$, and $\varepsilon_{o}$ is uniformly distributed in the range $[0,1)$. The carrier phase $\theta_{o}$ is generated as a uniformly distributed random variable in the 


$$
\begin{aligned}
\mathbb{E}\left[\Lambda\left(k_{1}\right) \Lambda\left(k_{2}\right)\right] & =\operatorname{tr}\left[\mathbf{B}_{k_{1}}^{T} \mathbf{R}_{\varepsilon_{o}}\right] \operatorname{tr}\left[\mathbf{B}_{k_{2}}^{T} \mathbf{R}_{\varepsilon_{o}}\right]+\operatorname{tr}\left[\mathbf{B}_{k_{1}}^{T} \mathbf{R}_{\varepsilon_{o}} \mathbf{B}_{k_{2}}^{T} \mathbf{R}_{\varepsilon_{o}}\right]+c\left(k_{1}, k_{2}\right), \\
\mathbb{E}\left[\Lambda\left(k_{1}\right) \Lambda^{*}\left(k_{2}\right)\right] & =\operatorname{tr}\left[\mathbf{B}_{k_{1}}^{T} \mathbf{R}_{\varepsilon_{o}}\right] \operatorname{tr}\left[\mathbf{B}_{k_{2}} \mathbf{R}_{\varepsilon_{o}}\right]+\operatorname{tr}\left[\mathbf{B}_{k_{1}}^{T} \mathbf{R}_{\varepsilon_{o}} \mathbf{B}_{k_{2}} \mathbf{R}_{\varepsilon_{o}}\right]+c\left(k_{1}, k_{2}\right),
\end{aligned}
$$

$$
c\left(k_{1}, k_{2}\right) \triangleq \frac{E_{s}^{2}}{T^{2}}\left(m_{4}-2\right) \sum_{n=-\infty}^{\infty}\left[\mathbf{a}_{n}\left(\varepsilon_{o}\right)^{H} \mathbf{B}_{k_{1}} \mathbf{a}_{n}\left(\varepsilon_{o}\right)\right]\left[\mathbf{a}_{n}\left(\varepsilon_{o}\right)^{H} \mathbf{B}_{k_{2}} \mathbf{a}_{n}\left(\varepsilon_{o}\right)\right]
$$

TABLE I

PARAMETERS OF DIFFERENT FEEDFORWARD TIMING ESTIMATION ALGORITHMS WHEN EXPRESSED IN THE FORM OF THE GENERAL ESTIMATOR

\begin{tabular}{|c|c|c|c|c|}
\hline & $Q$ & $\mathbf{B}_{k}$ & $K$ & remark \\
\hline Modified Lee's estimator [9] & 2 & eqn. (8) & 4 & - \\
Feedforward CML estimator [11] & 2 & eqn. (9) & 4 & $L_{g}=3$ \\
SLN estimator [1] & 4 & eqn. (5) & 4 & - \\
SLN estimator with pre-filter [5] & 4 & eqn. (13) & 4 & $h(n)=g(n T / Q) \cos (2 \pi n / Q)$ \\
& & & for $n=-5 Q, \ldots, 5 Q$ \\
\hline
\end{tabular}

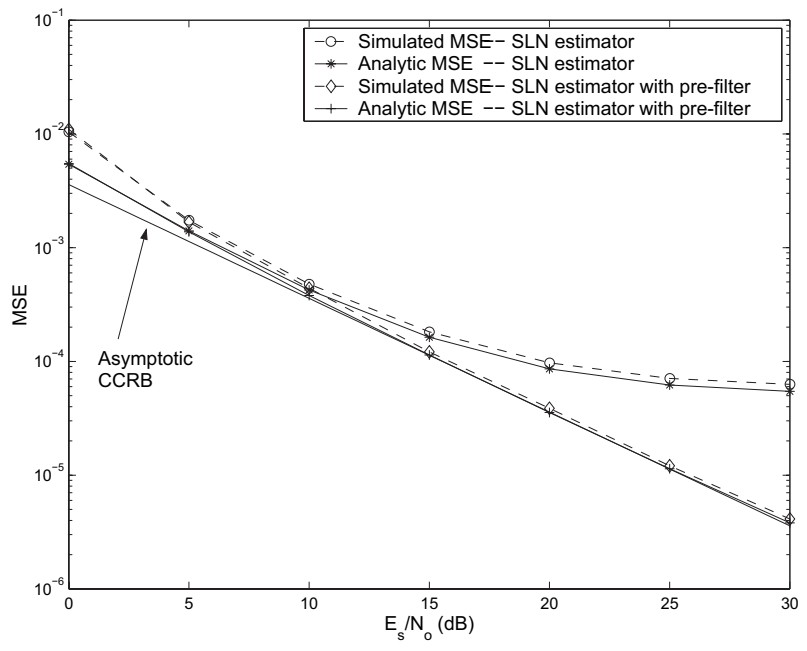

Fig. 3. Analytic and simulated MSEs for SLN estimator with and without pre-filter.

range $[-\pi, \pi)$, and assumed constant during each estimation. Each simulation point is obtained by averaging $10^{4}$ simulation runs. The asymptotic CCRB is computed assuming $Q=2$. In this letter, the results of the following representative estimators are presented:

1) Modified Lee's estimator [10]. The Lee's estimator is an algorithm obtained from an ad-hoc argument. Its modified version is used since it has a better performance than the original version.

2) Feedforward CML estimator [11]. This is an algorithm derived from a well-known statistical signal processing approach, namely the ML principle.

3) SLN estimator [1]. This estimator belongs to the class of cyclic correlation-based estimator (3). It is chosen because it was shown in [4] and [8] that the performance of (3) for $\tau=0$ appears to be the best.

4) SLN estimator with pre-filter [5]. This estimator is included to demonstrate the efficiency of pre-filter. The pre-filter used is $h(n)=\left.g(t) \cos (2 \pi t / T)\right|_{t=n T / Q}$ for $n=-5 Q, \ldots, 5 Q$ (i.e., $L_{p}=10 Q+1$ ) [5].
Notice that the first two estimators assume an oversampling ratio $Q=2$, while the last two estimators assume an oversampling ratio $Q=4$. The parameters for these four estimators when expressed in the form of the general estimator (2) are summarized in Table I.

For the computation of $\mathbf{B}_{k}^{C M L}$ and $\mathrm{CCRB}^{a s}\left(\varepsilon_{o}\right)$, there is a need to calculate $\varphi^{-1}$. Unfortunately, numerical calculations show that, for the $g_{r}(t)$ under consideration, $\varphi$ is not full rank (at least to the accuracy of Matlab). A main reason for rank deficiency is that, due to the nature of $g_{r}(t)$, when $|i-j|$ is large, the values of $[\varphi]_{i j}$ are very very small but not zero. A way to get around this is to replace $\varphi^{-1}$ by $\bar{\varphi}^{-1}$, where $[\overline{\boldsymbol{\varphi}}]_{i j}=[\boldsymbol{\varphi}]_{i j}$ if $|i-j|<L_{\varphi} Q$ and zero otherwise.

In this way, the matrix $\bar{\varphi}$ can be made full rank, but at the same time, significant part of the correlation between noise samples can still be represented accurately. Since most of the correlation induced by $g_{r}(t)$ is confined to a duration of a few symbols, $L_{\varphi}=4$ is used for the rest of the letter. Notice that the matrix $\varphi$ in $\mathbf{R}_{\varepsilon}$ need not to be replaced by $\bar{\varphi}$ since no inversion is required.

Fig. 2 shows the results for the modified Lee's estimator and the feedforward CML estimator. It can be seen that the analytical and simulation results match very well. Furthermore, the feedforward CML estimator performs much better than the modified Lee's estimator at high $E_{s} / N_{o}$ and its performance coincides with the asymptotic CCRB, meaning that the feedforward CML estimator is the best (in terms of MSE performance) within the class of symbol timing estimators employing second-order statistics. Fig. 3 shows the results for the SLN estimator with and without pre-filter. This figure also shows that the simulation results match the analytical results very well. Moreover, the figure shows that the application of a properly designed pre-filter removes the estimation error floor at high $E_{s} / N_{o}$ and makes the performance of the resultant estimator reaches the asymptotic CCRB.

\section{CONCLUSIONS}

In this letter, all the previously proposed feedforward symbol timing estimators employing second-order statistics were formulated into a unified framework. The finite sample mean square error (MSE) expression and the asymptotic conditional 
Cramer-Rao bound (CCRB) for this class of estimators were established. It was found that the analytical and simulation results match very well. Furthermore, it was found that the feedforward CML estimator [11] and the SLN estimator with a properly designed pre-filter [5] perform the best and their performances coincide with the asymptotic CCRB, which is the performance lower bound for the class of estimators under consideration.

\section{REFERENCES}

[1] M. Oerder and H. Meyr, "Digital filter and square timing recovery," IEEE Trans. Commun., vol. 36, pp. 605-612, May 1988.

[2] K. E. Scott and E. B. Olasz, "Simultaneous clock phase and frequency offset estimation," IEEE Trans. Commun., vol. 43, pp. 2263-2270, July 1995.

[3] F. Gini and G. B. Giannakis, "Frequency offset and symbol timing recovery in flat-fading channels: A cyclostationary approach," IEEE Trans. Commun., vol. 46, pp. 400-411, Mar. 1998.

[4] M. Ghogho, A. Swami, T. Durrani, "On blind carrier recovery in timeselective fading channels," in 33rd Asilomar Conf. Signals, Systems and Computers, vol. 1, 1999, pp.243-247.

[5] K. Shi, Y. Wang and E. Serpedin, "On the Design of Digital Blind Feedforward Jitter Free Timing Recovery Schemes for Linear Modulations," IEEE Trans on Commun., vol. 52, pp. 1464-1469, Sept. 2004.
[6] M. Morelli, A. N. D’Andrea and U. Mengali, "Feedforward ML-based timing estimation with PSK signals," IEEE Commun. Letters, vol. 1, pp. 80-82, May 1997.

[7] E. Panayirci and E. Y. Bar-Ness, "A new approach for evaluating the performance of a symbol timing recovery system employing a general type of nonlinearity," IEEE Trans. Commun., vol. 44, pp. 29-33, Jan. 1996.

[8] Y. Wang, E. Serpedin and P. Ciblat, "Blind feedforward cyclostationarity-based timing estimation for linear modulations," IEEE Trans. on Wireless Commun., vol. 3, pp. 709-715, May 2004.

[9] S. J. Lee, "A new non-data-aided feedforward symbol timing estimator using two samples per symbol," IEEE Commun. Letters, vol. 6, pp. 205207, May. 2002.

[10] Y. Wang, E. Serpedin and P. Ciblat, "An alternative blind feedforward symbol timing estimator using two samples per symbol," IEEE Trans. on Commun., vol. 51, pp. 1451-1455, Sept. 2003.

[11] Y.-C. Wu and E. Serpedin, "Design and analysis of feedforward symbol timing estimators based on the conditional maximum likelihood principle," IEEE Trans. on Signal Processing, accepted for publication, May 2004. Available online: http://ee.tamu.edu/ serpedin

[12] J. Riba, J. Sala and G. Vazquez, "Conditional maximum likelihood timing recovery: estimators and bounds," IEEE Tran. Signal Processing, Vol. 49, pp. 835-850, Apr. 2001. 